W. Kirchmann, Vereinfachte Darst. d. Nicotins, ev. auch Coniins ete. 209

Wie oben erwähnt, beträgt der Preis der drei Mittel : 30 , resp. $20 \mathrm{M}$.

Mit den Medicamenten folgt ein Brief, welcher in etwas unorthographischer Weise die Gebrauchsanweisung angiebt.

Herr Siggelkow sucht sich ausserdem durch seine „Wissenschaftliche Abhandlung über das menschliche Haar mit Bezug auf die Haarherstellungs-Präparate, " einem Büchelchen in gross Sedez mit 32 Seiten, Preis $40 \mathrm{Pf}$, bekannt zu machen. Das Werkchen warnt vor Universalmitteln, spricht über Zweck, Entstehung, Ergrauen und Ausfallen der Haare und empfiehlt schliesslich durch 31 Zeugnisse die Haarherstellungs - Präparate von Heinrich Siggelkow.

\title{
Vereinfachte Darstellung des Nicotins, ev. auch Coniins und Sparteins.
}

Von W. Kirchmann, Apotheker in Garding.

Ein mit zwei Gasleitungsröhren versehenes Blechgefäss wurde mit Tabak gefüllt, der zuvor mit einer Lauge von kohlensaurem Natron angefeuchtet war; das eine Glasrohr geht auf den Boden des Gefässes, das andere nur so weit, um den schliessenden Kork zu durchbohren. Mit Ausnahme der beiden Oeffnungen, welche durch die Gasleitungsröhren hervorgebracht sind, war das Gefäss, worin der Tabak war, gasdicht. Der Apparat wurde nun in ein siedendes Dampfbad gesetzt und das Rohr, welches bis auf den Boden des Gefässes ging, mit einem Kohlensäure-Entwicklungsapparat verbunden. Das zweite Ableitungsrohr wurde in ein Gemisch von Alkohol und verdünnter Schwefelsäure geleitet. Jetzt wurde ein rascher Strom Kohlensäure durch den Apparat geführt, und ergab dies Experiment eine reiche Ausbeute gänzlich farblosen Nicotins, an Schwefelsäure gebunden.

Um dies rein zu erhalten, versetzte ich die Lösung mit Aetzbaryt, verdampfte sie zur Trockne und zog das Nicotin mit Aether aus. -

Aroh. d. Pharm. IX. Bda. 9, Heft. 
210 E. Johansen, Beitr. z. Chemie d. Eichen -, Weiden- u. Ulmenrinde.

Eine Portion von saurer schwefelsaurer Nicotinlösung, die bereits recht concentrirt war, versetzte ich mit Thonerdehydrat, so viel sich lösen wollte. Alsbald erhielt ich schöne octaëdrische Krystalle, die ich für nichts Anderes halten kann, als für Nicotinalaun. Ich weiss zwar nicht, ob schon ein Fall bekannt ist, wo eine tertiäre Diaminbase die Stelle des Ammoniaks im Alaun ausfiullt.

Jedenfalls wäre dies eine Art, um Nicotin in recht bequemen Krystallen zu erhalten und gäbe eine Garantie, um dasselbe rein darstellen zu können.

\section{Mittheilungen aus dem pharmaceutischen Institute zu Dorpat.}

Beiträge zur Chemie der Eichen-, Weiden- und Ulmenrinde.

Von Mag. Edwin Johansen, Assistent am pharmaceut. Institut zu Dorpat.

So bequem auch Wagner's*) Annabme eines „physiologischen und pathologischen Gerbstoffs" erscheint, so ist diese doch wiederholt als nicht stichhaltig durch die Arbeiten von Stenhouse **), Löwe ${ }^{* * *}$ ), Rembold $\dagger$ ), Hlasiwetz $\dagger \dagger$ ), Günther $+\dagger \dagger$ ) u. A. erkannt worden. Es ist auch dargethan, dass der Gerbstoff der Sumachblätter, Dividivi, Myrobalanen etc. die grösste Aehnlichkeit mit der Gallusgerbsäure besitzt. Immerhin ist aber die Frage nach den chemischen Verhältnissen noch für viele, namentlich auch zur technischen Ver-

*) Ztschrft. für anal. Chem. v. Fresenius. Jahrg. 5. p. 1.1866. und Polytechn. Journ. v. Dingler. Bd. 1. pag. 3. 1867.

**) Annal. d. Ch. und Pharm. 1843. Bd. 45. pag. 9. 15. 16. 17.

***) Ztsehrft f. anal. Ch. Jahrg. 12. 1873. p. 128.

†) Annal. d. Ch. u. Pharm. Bd. 143. p. 286.1867.

$\dagger+)$ Ebendas. Bd. 142. p. 234.1867.

$\dagger+\dagger)$ Beiträge zur Kenntniss der im Sumach, in den Myrobalanen u. der Dividivi vorkommenden Gerbsäuren. Inaugural-Dissert. Dorpat 1871. 